Archives

11 | 1993

Histoire intellectuelle // Réflexions collectives sur

l'histoire sociale

\title{
Persistances et conservatismes dans les connaissances cosmographiques à la Renaissance
}

Corrado Vivanti

\section{(2) OpenEdition \\ 1 Journals}

Édition électronique

URL : http://journals.openedition.org/ccrh/2770

DOI : $10.4000 /$ ccrh. 2770

ISSN : $1760-7906$

Éditeur

Centre de recherches historiques - EHESS

Édition imprimée

Date de publication : 10 octobre 1993

ISSN : 0990-9141

Référence électronique

Corrado Vivanti, «Persistances et conservatismes dans les connaissances cosmographiques à la Renaissance », Les Cahiers du Centre de Recherches Historiques [En ligne], 11 | 1993, mis en ligne le 05 mars 2009, consulté le 20 avril 2019. URL : http://journals.openedition.org/ccrh/2770 ; DOI : 10.4000/ ccrh. 2770

Ce document a été généré automatiquement le 20 avril 2019.

Article L.111-1 du Code de la propriété intellectuelle. 


\title{
Persistances et conservatismes dans les connaissances cosmographiques à la Renaissance
}

\author{
Corrado Vivanti
}

\section{NOTE DE L'AUTEUR}

Conférence prononcée au séminaire de Jacques Revel le 26 mars 1993.

1 Au début de l'année 1543, l'imprimeur parisien Guillaume Richard publiait une nouvelle édition du traité De Sphaera de John of Holiwood, le savant mathématicien du XIII ${ }^{\mathrm{e}}$ siècle, mieux connu sous son nom latinisé de Sacrobosco. Dans la longue histoire de cet ouvrage, cette édition pourrait bien marquer un tournant significatif.

Dès sa parution, le petit traité de Sacrobosco avait connu un succès extraordinaire: répandu par un nombre considérable de manuscrits, il eut, après l'édition princeps de Ferrare en 1472, des dizaines d'éditions et fut traduit en Italie et en France, en Angleterre et en Allemagne, en Pologne et en Espagne. En 1650, il était encore publié à Madrid. Si sa concision et sa clarté peuvent expliquer sa fortune, il offrait surtout aux adversaires de la théorie copemicienne, dans la seconde moitié du XVI ${ }^{\mathrm{e}}$ siècle, des arguments en faveur de la théorie ptolémaïque. Ainsi, en 1570, c'est dans son commentaire du De Sphaera que le jésuite Clavius réfutait l'hypothèse de l'astronome polonais. Il faut ajouter que plus tard, en 1611 - il avait connu Galilée entre-temps -, il concédait, dans la dernière édition, qu'il était nécessaire de trouver un nouveau système mieux capable de s'accorder avec les récentes découvertes, dès lors que l'ancien n'arrivait plus à les expliquer de manière convaincante.

3 La publication de 1543 excite notre curiosité à cause de sa préface. L'auteur en était Philippe Mélanchton, et il est sans doute curieux de voir le nom de l'ami et plus proche collaborateur de Luther au frontispice d'un livre imprimé à Paris. Il ne faut pas oublier en 
effet que, neuf ans auparavant, on y avait assisté, non sans émotion, à la procession solennelle que le roi en personne avait organisée en signe d'expiation du crime perpétré par les hérétiques, qui avaient recouvert la France de placards contre la messe, jusque dans le cabinet même de François $\mathrm{I}^{\mathrm{er}}$. Telle était néanmoins l'autorité intellectuelle du praeceptor Germaniae qu'il était à même de faire oublier ses opinions religieuses discutables. Dans les années suivantes encore, certains de ses écrits furent publiés dans le royaume du roi Très-Chrétien.

4 A propos de l'édition parisienne, on peut se demander si elle n'était pas destinée à contrecarrer les théories coperniciennes. Au premier abord, on pourrait en douter : 1543 étant justement l'année de publication du De revolutionibus orbium coelestium, il serait trop tôt pour polémiquer avec le nouveau système. D'autant plus que le texte de Melanchton, utilisé comme préface, était en réalité une lettre adressée deux ans auparavant au théologien et humaniste souabe Simon Grynaeus (Griner). Toutefois, les milieux savants avaient déjà entendu parler des théories coperniciennes bien avant la parution du $D e$ revolutionibus : en 1539, Georges Rhéticus, jeune professeur de l'Université de Wittenberg dirigée par Melanchton, s'était rendu à Frauenburg, en Prusse Orientale, afin de rencontrer Copernic et d'obtenir des éclaircissements sur ses théories cosmologiques dont il avait eu vent. Peu après son arrivée, il écrivait une lettre enthousiaste à Johann Schoener, professeur de mathématiques au Gymnasium de Nuremberg, et l'année suivante, il lui dédiait sa Narratio prima, la première version imprimée de la nouvelle astronomie. Melanchton reçut de Rhéticus lui-même une copie de cet opuscule et sa réaction fut nettement défavorable, puisqu'il déclara qu'aucun gouvernement ne devrait tolérer la diffusion d'idées aussi absurdes.

Les yeux nous sont témoins, écrira-t-il dans les Initia doctrinae physicae, que les cieux achèvent une révolution dans l'espace de vingt quatre heures. Il y a des hommes, toutefois, qui pour l'amour des nouveautés ou pour faire preuve d'ingéniosité ont établi que la Terre se meut, et ils prétendent qu'aussi bien la huitième Sphère que le Soleil ne tournent pas... Eh bien, soutenir des conceptions pareilles, c'est un manque d'honnêteté et de dignité, et cet exemple est dangereux. C'est le devoir de tout esprit sain d'accepter la vérité telle qu'elle nous a été révélée par Dieu et de se soumettre à elle.

Il n'est donc pas étonnant qu'à son retour à Wittenberg au second semestre universitaire de 1540, Rhéticus fût obligé par Mélanchton, à qui revenait la responsabilité d'établir le programme des cours, de consacrer ses leçons justement au traité de Sacrobosco.

Il est bien possible, partant, que la lettre de Mélanchton, autant que son utilisation pour l'édition parisienne de 1543, aient été une sorte de mesure préalable destinée à barrer le chemin de la France aux théories coperniciennes. En tout cas, le texte du réformateur allemand nous apparaît comme une apologie du vieux traité, exalté précisément à cause de son ancienneté. S'il avait été reçu comme un ouvrage utile pendant tant de siècles, soutenait Melanchton, cela voulait dire qu'il l'était effectivement: "Cum autem hic libellus tot seculis, in omnibus scholis, in tanta varietate iudiciorium, genios habuerit propitios, necesse est eum rebus optimis refertum esse. » Ce traité avait su résumer d'une façon claire la «sphaerica scientia » et avait jeté les bases de tout développement du sujet. D'après Melanchton, Sacrobosco, « ex tanta tamque multiplici rerum copia », avait réussi à « eligere ea quae maxima in aditu ac vestibulo profutura essent ». C'est pour cette raison, concluait-il, que « semper amavi mirifice libellum Joannis de Sacrobosco ».

7 En effet, c'est bien la «sphaerica scientia » qui permet de comprendre les mouvements des astres et leur influence sur la vie des hommes. Le fidèle chrétien ne doit pas craindre 
cette connaissance, au contraire, puisqu'elle lui donne la possibilité de saisir « certam rationem divinitus ostensam », un ordre certain déployé par la volonté divine. L'étude des astres peut nous éclaircir le rapport entre le Ciel et les hommes. Que Melanchton, le savant et pieux humaniste, ait attribué, à la différence de Luther, tant d'importance à l'astrologie, n'est pas surprenant pour nous qui connaissons - grâce à l'étude d'Aby Warburg - son souci de tirer l'horoscope de son maitre, allant jusqu'à altérer sa date de naissance pour l'accorder avec le déclenchement de la Réforme. L'astrologie n'était pour lui qu'une partie de la physique céleste, et ne portait donc aucune atteinte à la foi. C'était plutôt ceux qu'il appelait «nonnulli Epicurei theologi » qui, se moquant de cette branche du savoir, devaient être critiqués et désavoués. Pour sa part, déclarait-il solennellement, personne n'aurait pu le convaincre que les étoiles n'avaient pas d'influence sur les éléments et sur les êtres animés. Aristote n'avait-il pas affirmé que le monde inférieur est gouverné par le monde supérieur et que les choses supérieures sont la cause première du mouvement des corps inférieurs?

Ce partisan convaincu de la théorie ptolémaïque ne nourrissait d'ailleurs aucun doute sur ce que Sacrabosco expliquait, conformément à la doctrine de la Scholastique : «Sphaera Mundi dividitur secundum substantiam et secundum accidens ", et encore, selon la substance, elle se divise "in spaeras novem ", c'est-à-dire "in sphaeram quae primus motus sive primum mobile dicitur, et in sphaeram stellarum fixarum quae firmamentum nuncupatur, et in septem sphaeras septem planetarum. » La Terre, formant avec l'eau un seul globe, était le « centrum mundi », soit la sphère placée au milieu de l'univers.

9 N'aurions-nous à faire qu'à une résistance entêtée aux nouveautés cosmologiques qui semblaient menacer la vision judéo-chrétienne du monde? Pas uniquement. En effet, Sacrobosco décrit la Terre non seulement comme la sphère placée au centre de l'univers, mais aussi comme un globe divisé en cinq zones, dont deux sont habitables : les médianes, situées entre l'extrême rigueur des froids polaires et l'insupportable chaleur équatoriale. Pour l'expliquer, il invoquait les vers d'Ovide, l'une des sources les plus vénérées des savants du Moyen Age :

Utque duae dextra caelum totidemque sinistra

parte secant zonae, quinta est ardentior illis,

sic onus inclusum numero distinxit eodem

cura dei, totidemque plagae tellure premuntur.

Quarum quae media est, non est habitabilis aestu ;

nix tegit alta duas : totidem inter utramque locavit

temperiemque dedit mixta cum frigore flamma ${ }^{1}$.

Cette description était acceptable au XIII ${ }^{\mathrm{e}}$ siècle, alors qu'on n'avait qu'une idée imparfaite du monde. Il nous parait pour le moins invraisemblable qu'Ovide fût encore une autorité en matière de géographie trois siècles plus tard, après les voyages de découverte et la publication de cartes qui relevaient les terres nouvelles. Melanchton, en présentant le traité De Sphaera, n'éprouvait pourtant nul besoin d'informer les lecteurs, qu'au moins pour ce qui concernait la description de la Terre, l'ouvrage de Sacrobosco ne répondait plus à la réalité connue, surtout s'agissant de l'impossibilité d'habiter les terres et même de naviguer sur les mers situées au-dessous du vingtième degré " propter calorem solis » et au nord de l'Ecosse " propter frigorum asperitatem ».

11 Devons-nous penser que Melanchton ne se soucia pas de préciser des notions qu'il pouvait considérer comme acquises? Je crois plutôt que ce détail ne lui paraissait pas important ; malgré les nouveautés qui avaient transformé la connaissance du monde, de nombreux savants n'en avaient pas tout à fait saisi toute la portée, à savoir la rupture 
radicale avec la science traditionnelle. Le cas de Melanchton qui, ne l'oublions pas, dirigeait la plus importante université allemande de l'épopque, n'est pas unique. Tout au long $\mathrm{du} \mathrm{xVI}^{\mathrm{e}}$ siècle, nous pouvons suivre l'extraordinaire fortune d'une ouvrage encyclopédique intitulé Margarita philosophica, dont l'auteur était un chartreux vivant à la cour de l'empereur Maximilien $\mathrm{I}^{\mathrm{er}}$ : Gregor Reisch. En 1599, une traduction italienne en était encore publiée à Venise. Cette encyclopédie, composée à la fin du $\mathrm{Xv}^{\mathrm{e}}$ siècle mais publiée en 1503, donnait de la Terre une définition inspirée par celle de Sacrobosco: « Terra, una cum aqua, [...] constituens sphaeram, in superficie concava aeris continetur et tamquam centrum in medio mundi consistit. » Et pour Reisch aussi, la Terre était partagée, malgré les navigations ibériques, en cinq zones dont trois infranchissables à cause du froid ou de la chaleur. De régions hospitalières, il ne comptait que l'Europe, l'Asie et l'Afrique et ne mentionnait donc pas les Amériques, pas même dans les éditions « castigatae et emendatae » du XvI siècle avancé. Ni l'auteur - encore vivant lorsque des informations sûres concernant le Nouveau Monde devinrent disponibles - ni ses nombreux lecteurs ne se posèrent, semble-t-il, la question de la conciliation des vieilles conceptions du monde avec les connaissances nouvelles.

Nous trouvons par contre une indication curieuse : «Est in Terra, ut aiunt, infernus quadruplex ", il y avait quelque part sur Terre, disait-on, vraisemblablement dans l'hémisphère méridional, un enfer divisé en quatre sections, superposées les unes sur les autres, recelant l'enfer proprement dit, deux limbes différents et le purgatoire. On en était donc encore à la cosmographie de Dante Alighieri.

Il y avait ainsi une continuité bien établie de la vision à la description du monde, que les découvertes n'avaient pas compromise. Il suffit de consulter, pour s'en rendre compte, dans les éditions de la Géographie de Ptolémée publiées au cours du Xvie siècle, les cartes probablement dessinées par de doctes byzantins trois siècles auparavant. Cet ouvrage du savant d'Alexandrie continua d'être publié et comsulté comme atlas géographique, tout au mieux avec l'addition de cartes nouvelles qui ne remplaçaient pas les anciennes mais se juxtaposaient à elles. La persistance de ces cartes ne peut pas manquer de nous surprendre, étant données les erreurs désormais établies et la disponibilité de nouvelles cartes mise en appendice. Il est vrai que le mérite de Ptolémée était d'avoir construit ses cartes suivant la méthode mathématique, de sorte que son enseignement était précieux pour des hommes accoutumés à une cartographie sans précision dans la détermination de l'espace. Ptolémée - avait déjà remarqué un savant de la première moitié du $\mathrm{xv}^{\mathrm{e}}$ siècle - traite des choses, c'est-à-dire des lieux de ce monde, en calculant leur distance, en largeur et en longueur, du cercle équatorial.» Cela n'empêche que ses cartes représentaient les terres et les mers autrement que les nouvelles connaissances le préconisaient. On a observé que « la présence dans les Atlas de Ptolémée des différentes Italia, Gallia, Ispania nova à côté des cartes de Ptolémée des mêmes pays, fréquente dès le milieu du $\mathrm{Xv}^{\mathrm{e}}$ siècle, montre que les géographes, ayant reconnu les mérites et les défauts des deux types de cartes, les rapprochaient, en attendant que le travail de contrôle et de correction arrivât à son terme ». Il y a, dans le savoir du XVI ${ }^{e}$ siècle, une vaste région d'incertitute ou même d'indifférence. Il s'agit d'un problème qui, avant l'histoire des sciences, paraît concerner l'histoire des mentalités au début de l'âge moderne, et qu'il faudrait aborder par une recherche vaste et sérieuse sur les textes et les manuels de cosmologie et de cosmographie, mais aussi par l'analyse des traités de morale ou bien des correspondances, qui permettent de discerner les différentes façons de penser courantes à l'époque. Cette étude pourrait nous faire comprendre l'attitude et même les doctrines 
scientifiques de ces siècles. Que l'on songe au fait que Copernic n'arriva pas à éliminer de son système ni les orbes, c'est-à-dire les sphères cristallines où les planètes sont enchâssées, ni le principe du cosmos fini et clos. Il faudra attendre Giordano Bruno pour entendre parler De l'infinito universo e mondi. Mais il s'agit là d'un hérétique, et qui finit sur le bûcher.

Nous sommes alors amenés à présumer que les mêmes oscillations, les mêmes marges d'imprécision dans le savoir, reviennent en général dans la vie intellectuelle et spirituelle de ce temps, et qu'elles s'approchent de certaines formes de pensée qui tendent à repousser aussi bien les innovations les plus radicales que les choix définitifs. Ainsi pourrions-nous comparer le traditionalisme de Melanchton, dont témoigne sa réaction à l'annonce de la théorie copernicienne, à sa remise en selle de la patrologie. Le retour aux Evangiles ne lui paraît plus suffisant pour bâtir la nouvelle Eglise réformée : il lui est nécessaire de recourir aux textes des premiers siècles de la chrétienté. Dans ce cas aussi l'appel à la « sainte Antiquité » prend une double signification, celle d'un étai du moderne établi mais en même temps celle d'un frein et d'un fondement pour la restauration de valeurs traditionnelles. Ce que nous appellerions aujourd'hui une "normalisation ».

intéressant de considérer sous ce jour d'autres cas contemporains, comme la théorie de la double vérité, ou bien cet indifférentisme religieux qu'était pour les réformateurs le nicomédisme, c'est-à-dire la tendance à ne pas se prononcer ouvertement, dans les pays catholiques, pour la Réforme, tout en en partageant les exigences et les principes; ou encore l'irénisme et sa recherche des fundamentalia fidei, les principes communs à tous les chrétiens, afin de parvenir à la paix religieuse. A cet égard, le marranisme aussi, ou bien la doctrine de la "dissimulation honnête ", devraient aussi être pris en considération. Il s'agit, bien entendu, de tendances et d'attitudes que provoqua, avant tout, la répression organisée par les institutions dominantes; et néanmoins, il faudrait les considérer aussi comme les produits d'incertitudes et d'ambiguïtés qui s'imposent dans les milieux intellectuels depuis que des points de repère traditionnels sont venus à manquer et les autorités culturelles du passé sont en crise.

Nous risquons de tomber dans l'anachronisme - le péché mortel des historiens disait Lucien Febvre - si nous essayons de distingueer trop nettement les antinomies de la recherche scientifique et les incertitudes ou certaines aversions de la sensibilité religieuse, puisqu'il n'y avait aucune séparation - nous le savons bien - entre la conception du cosmos, la vision du monde naturel et le respect des préceptes de la foi. Il y a des moments dans la connaissance, distincts et d'une certaine manière parallèles, qu'il serait faux de juger d'un point de vue de moraliste ou de considérer comme dépourvus de rationalité. Quand Copernic, dans sa lettre de dédicace au pape Paul III, soutient que sa théorie ne porte pas atteinte à la foi parce que « mathemata mathematicis scribuntur ", il ne veut pas fournir une justification opportuniste, même s'il se doute bien du danger que représente sa doctrine, sinon il n'aurait pas gardé le secret sur son ouvrage pendant des décennies. Et c'est la même façon de penser qui conduit Galilée à abandonner ses expériences de physique sur la chute des corps que son ami et collaborateur Paolo Sarpi jugeait capables d'assener un coup mortel à l'aristotélisme et par conséquent à l'Eglise de la Contre-Réforme, pour s'adonner entièrement à l'astronomie qu'il croyait, jusqu'à la veille du procès de 1616, acceptable pour l'Eglise. Peut-être sans ces apories de la connaissance, le monde savant n'aurait-il pas emprunté ces chemins conduisant à la crise de la conscience européenne? 
17 La ténacité avec laquelle les publications que je viens de mentionner continuaient à proposer une géographie dépassée nous paraît d'autant plus étonnante qu'au $\mathrm{XV}^{\mathrm{e}}$ siècle, la division de la Terre en zones avait souvent été passée sous silence, voire même désavouée par des savants qui tenaient compte des voyages portugais le long des côtes africaines. Partant, il ne me semble pas possible de dresser un tableau d'ensemble des connaissances cosmographiques de l'époque et d'établir le moment où s'effectue le passage de l'ancienne à la nouvelle conception du monde. Je dois dire en effet, que l'essai de W.G. Randles, De la Terre plate au globe terrestre, tout en étant le fruit d'une recherche rigoureuse, ne me convainc pas tout à fait, le sous-titre, en particulier, me paraît raidir sa thèse, en évoquant une "mutation rapide" à placer entre 1480 et 1520 . Le lecteur pourrait être amené à croire que, jusqu'en 1480, la doctrine de la Terre plate était généralement acceptée et qu'en l'espace de quarante ans, la notion de sphéricité de la Terre avait triomphé. Or, quant à celle-ci, nous avons déjà vu quel retard la persistance des anciennes croyances lui ont imposé ; pour ce qui concerne la platitude terrestre, il suffit de se rappeler qu'au XIII ${ }^{\mathrm{e}}$ siècle, un penseur comme Albert le Grand, le maître de Saint Thomas d'Aquin, traita de "vulgaris imperitia ", de "sottise populaire ", l'opinion que la zone torride n'était pas habitable et que ceux qui ont les pieds tournés vers nous, c'est-à-dire ceux qui se trouvent aux antipodes, devaient tomber hors de la Terre.

A vrai dire, sur tous ces problèmes, nous trouvons, au cours de ces siècles, des contradictions et des oscillations. Il y a des positions encore proches de la Topographia christiana de Côme Indopleuste, le savant byzantin qui, à l'époque de Justinien, avait résumé les opinions des Pères de l'Eglise qui décrivaient la Terre comme un parallélogramme contourné par l'Océan et découpé par quatre golfes disposés en forme de croix ; tandis que d'autres savants - et c'est justement le cas de Sacrobosco - n'ont pas de doutes sur la sphéricité de la Terre. En tout cas, au début du XV ${ }^{\mathrm{e}}$ siècle, la Géographie de Ptolémée, attribuant à la Terre une forme sphérique, parvint en Occident et fut traduite en latin par Jacopo d'Angiolo. La réception des savants fut enthousiaste et, avec l'invention de l'imprimerie, sept éditions parurent avant 1500.

19 L'une des premières, dédiée au pape Paul II en 1478, était préfacée par l'ancien secrétaire général du cardinal Bessarion, l'humaniste de Vérone Domizio Calderini. Celui-ci, à propos de la cosmologie de l'astronome d'Alexandrie, remarquait qu'une entreprise de longue haleine avait été requise pour arriver à établir que « universam terram esse totius mundi urbiculare centrum ». Il faisait donc l'éloge de Ptolémée pour avoir su rassembler, choisir et ordonner toutes les données accumulées par différents auteurs de l'Antiquité, en réussissant à présenter une vision d'ensemble de la Terre "in unum quasi corpus ». Calderini avait pris sur lui de réviser le texte grec et de collationner les manuscrits latins "cum vetustissimo graeco manu Gemisti philosophi », tandis que l'allemand Conrad Sweinheim, "a quo formandorum Romae librorum ars primum profecta est », devait imprimer l'ouvrage et graver des plaques de cuivre pour les cartes. Mais, après avoir travaillé sans cesse pendant trois ans, il était malheureusement mort et un autre allemand, "non inferiori ingenio ac studio », avait pris sa place : il s'agissait d'Arnold Buckinck, qui avait mené l'entreprise à bonne fin. Tous les passages d'un travail d'édition accompli conformément aux règles de la philologie humaniste sont ainsi rappelés et Calderini conclut en soulignant le lien étroit qui doit être établi, pour une juste connaissance des choses, entre la géographie et l'astronomie. La capacité de Ptolémée de nous faire connaître "divinas astrorum terraeque lucubrationes » constituait son plus 
grand mérite : il avait réussi à joindre, grâce à ses calculs, «huius terrae situs et loca » avec « caeli singulis artibus».

Nous savons que, par la suite, les vieilles cartes furent souvent corrigées grâce aux connaissances dérivées des portulans ou des croquis de voyageurs, et en général la représentation cartographique s'améliora de plus en plus. Il faut dire que la géographie connut au $\mathrm{XV}^{\mathrm{e}}$ siècle un essor qu'Alexandre Humboldt a ainsi décrit dans son Cosmos:

Le $\mathrm{xv}^{\mathrm{e}}$ siècle appartient à ces rares époques dans lesquelles tous les efforts intellectuels offrent le caractère commun d'une tendance invariable vers un but déterminé. L'unité des efforts, le succès qui les a couronnés, l'active énergie que manifestèrent des peuples entiers donnent à l'âge de Colomb, de Sébastien Cabot et de Gama un éclat brillant et durable. "C'est pour cela qu'il remarque ensuite : « La découverte des régions tropicales de l'Amérique par Christophe Colomb, Alfonso de Hojeda et Alvarez Cabral ne peut être considérée comme un événement isolé dans l'histoire de la contemplation du monde.

Elle doit être considérée en liaison avec tout l'effort culturel qui la précède. Sous ce rapport, il faudra souligner l'importance que deux grandes synthèses cosmographiques - l'Ymago Mundi de Pierre d'Ailly et la Historia rerum ubique gestarum du pape Pie II eurent pour Colomb. Nous avons la possibilité de lire l'oeuvre du cardinal de Cambray avec les apostilles que Colomb y apporta, grâce à l'édition de l'Ymago Mundi donnée par Edmond Buron en 1930. Par contre, il faut déplorer que la Historia du savant humaniste monté sur le trône pontifical n'ait jamais eu une édition moderne enrichie par les annotations du découvreur génois. Les deux ouvrages furent pour colomb une stimulation et une aide, et ses annotations nous font saisir l'effort intellectuel qui lui fut nécessaire pour en extraire un fondement scientifique valable de ses desseins. Sans doute, Coulomb n'était-il pas le découvreur qu'une historiographie hypercritique autant qu'ingénue nous a dépeint sous les traits d'un navigateur qui, ayant de la chance, pouvait bien se passer de toute préparation intellectuelle. Peut-être n'était-il même pas le savant accompli qu'il s'imaginait être dans cette lettre au roi d'Espagne en 1501.

" Dès mon premier âge ", écrivait-il, «j'ai commencé à naviguer et aujourd'hui encore je vais par mer. C'est cet art qui pousse celui qui le pratique à aspirer à la connaissance des secrets de ce monde. A présent, je le pratique depuis quarante ans et j'ai parcouru toutes les routes connues. J'ai étudié et j'ai eu des conversations avec des savants, aussi bien ecclésiastiques que séculiers, latins et grecs, juifs et mauresques, ainsi qu'avec d'autres personnes appartenant à des sectes différentes. Notre Seigneur s'est montré très généreux en me donnant cette attitude et je tiens de Lui un esprit intelligent. Il me fit expert en marine, Il m'a pourvu, autant qu'il le fallait, en astrologie, en géométrie et en arithmétique, Il m'a donné l'adresse de dessiner la sphère avec ses villes, ses fleuves, ses montagnes, ses îles, ses ports, chaque chose à sa place. En ce temps-là, j'ai vu et je me suis efforcé d'étudier tous les livres de philosophie et des autres arts... »

Toutefois, entre ces deux images, il y a place pour une troisième possibilité, tout en se rappelant que ce qu'il écrivait de lui-même n'était pas simple vantardise. Ce sont justement ses apostilles en marge de plusieurs classiques qui en apportent le témoignage. Je n'entrerai pas dans la question compliquée de leur authenticité : je me borne à signaler que la critique la plus récente tend à conclure que, quand bien même elles ne seraient pas toutes de la main de Colomb, c'est lui qui les a voulues et qui les a organisées.

24 Naturellement, pour que la lecture des ouvrages de Pierre d'Ailly et de Pie II provoquât des résultats si exceptionnels, il fallait un lecteur lui-même hors du commun. Nous 
pourrions comparer le résultat de ces lectures de Colomb à celui que Copernic tira des textes préptolémaïques, à savoir la formulation de son hypothèse héliocentrique. Cette comparaison nous amène à une autre remarque : en général, c'est l'autorité des textes qui prime alors l'expérience directe. C'était presque inévitable pour Copernic, alors que l'observation astronomique se faisait encore à l'oeil nu. Dans le cas des auteurs étudiés par Colomb, la situation est différente. Par exemple, nous savons que qu'Enea Silvio fit personnellement l'expérience d'une traversée - 0 combien hasardeuse ! - de la Norvège à l'Ecosse du Nord, où il parvint «brumali tempore... cum sol paulo amplius quam tres horas terram illuminaret ». Il connaissait aussi, pour en avoir entendu parler dans ces pays, l'existence des îles Orcades, de l'islande et de terres encore plus proches du pôle. Et néanmoins, de cela, il ne nous dit rien dans son Historia. Quand, dans un chapitre de cet ouvrage, il se demande s'il est possible de naviguer sur certaines mers, il répond par l'affirmative quant à la zone torride, en citant Pline et Annon carthaginois, mais pour la zone septentrionale - celle justement dont il avait l'expérience - il paraît incertain : « De septentrionali oceano maior contentio est ", écrit-il, et il conclut toujours sur la foi des classiques, que là aussi la navigation semble possible.

Par contre, Colomb n'hésite pas à souligner la valeur des expériences faites à son époque par les navigateurs qui l'ont précédé. Peut-être mettait-il à profit l'avertissement de Ptolémée, qui avait écrit au début de sa Géographie que c'est surtout le récit des voyages et des navigations d'autrui qui peut guider dans l'intelligence de cette science. Ainsi, dans son apostille au passage où Pie II avait rapporté l'opinion de Parménide sur la division du Ciel et de la Terre en cinq zones, Colomb note: «Contrarium probatur in austro per Portugalenses et in septentrione per Anglos et Suevos, qui eas partes navigant. » Encore : le pape humaniste paraissait enclin à penser que - contrairement à l'opinion de ceux qui avaient soutenu que l'Océan Indien est une mer fermée, sans aucun passage vers l'Atlantique - l'Afrique était circumnavigable, et il citait à preuve l'avis de l'ancien géographe Solinus, pour qui « quod ab India usque in Hispaniam et dorsum Africae sit navigandum ». Mais Colomb procède de manière expéditive, en rappelant qu'« in Arabico sinu signa navium ex hispaniensibus naufragiis » avaient été trouvés plus d'une fois.

D'ailleurs, c'est justement à cause de l'importance conférée par la culture officielle aux textes écrits que nous pouvons comprendre le désir de Colomb d'y trouver un appui de ses hypothèses. Il ne s'agissait pas seulement de l'aspiration à une certitude théorique : avant qu'Isabelle lui donnât les trois fameuses caravelles, les rois Catholiques consultèrent plus d'une fois les théologiens. Non seulement parce qu'ils avaient la réputation d'être détenteurs de la suprême sagesse, mais aussi parce qu'il y avait à décider en matière de foi. En effet, d'après une opinion accréditée, la Bible aurait attribué à la Terre émergée, ou du moins à sa partie habitée, des dimensions tellement réduites qu'elle n'aurait été qu'une île, pratiquement plate, perdue dans l'Océan. En outre, du récit de la création dans la Genèse et du psaume 104, plusieurs interprètes avaient déduit que Dieu avait rassemblé les eaux dans un seul endroit. Il y avait bien sûr des mers intérieures, mais c'était l'Océan qui entourait l'écoumène en recouvrant les trois quarts de la surface du globe. Ainsi la proposition avancée par Coulomb - de pouvoir atteindre le Levant en naviguant vers le Couchant - parut à plusieurs comme blasphématoire bien plus que sans fondement. Saint Augustin n'avait-il pas dit: "Maior est Scripturae auctoritas quam omnis humani ingenii capacitas »? et à Saint Augustin, en niait l'existence. Encore en 1492, quand les rois Catholiques, sur le 
point d'attaquer Grenade, convoquèrent un conseil de théologiens, plusieurs d'entre eux déclarèrent hérétique une doctrine qui présupposait l'existence des antipodes, et ils furent en mesure de citer non seulement les pères de l'Eglise, mais aussi Nicolas de Lire, le savant fanciscain du $\mathrm{XIV}^{\mathrm{e}}$ siècle, pour lequel la Terre «nulla latera habere per inferiorem partem sphaerae obtorta ». Cependant, la question la plus grave, plus brûlante encore que celle de la forme terrestre, consistait dans l'existence présumée de populations que la Révélation n'avait pas atteintes, ce qui paraissait contredire l'universalité de la prédication du Christ et des apôtres. En rapportant le quatrième verset du psaume 19, Saint Paul avait écrit aux Romains (10.12) : « In omnem terram exivit sonus eorum ", c'est-à-dire des apôtres. Comment résoudre le problème? Certes, des esprits désinvoltes comme Luigi Pulci, qui vécut à la cour de Laurent de Médicis et qui fut l'auteur d'un poème chevaleresque Morgante maggiore avaient enterré la question, bien avant le voyage de Colomb, par le relativisme religieux. Dans ce poème, le savant diable Astarotte, ayant expliqué à Renaud qu'il était possible d'aller dans l'autre hémisphère, aux pays des antipodes, et qu'il y avait là aussi des villes, des châteaux, des empires et des terres peuplées, avait dû répondre à l'objection du paladin : « Si ces gens-là sont eux aussi descendus d'Adam, comment peuvent-ils atteindre le salut? » Il avait répliqué qu'il suffit d'observer sa propre loi avec rectitude pour ne pas devoir désespérer de la récompense divine (XXVI, 228-36). Mais ce ne fut pas un hasard si, en 1484, le poète fut enseveli en terre déconsacrée en tant qu'hérétique.

Le problème se posa naturellement de manière plus éclatante après la découverte de l'Amérique. C'est Guichardin qui nous le rappelle dans son Histoire d'Italie: "A cause de ces navigations [des portugais et des espagnols] il est devenu manifeste que les anciens s'étaient trompés sur différents points regardant la connaissance de la Terre. » En plus du fait que la théorie des cinq zones s'avérait sans fondement, il fut bientôt clair que d'autres hommes, nommés "antipodes», vivaient "sous nos pieds». Et il continue: "Non seulement ces navigations ont confondu beaucoup de choses que les écrivains des choses terrestres ont affirmées, mais elles ont donné de l'anxiété aussi aux interprètes de la Sainte Ecriture, qui avaient l'habitude d'expliquer le verset du psaume: de la terre tout entière sortit leur son et leur parole arriva jusqu'au bout du monde, comme s'il avait affirmé que la foi du Christ avait pénétré partout dans le monde, grâce à la bouche des apôtres. »

Il ne fut pas facile de se convaincre que " cette interprétation était contraire à la vérité ", même s'il n'était " pas possible de croire - n'ayant jamais eu aucune nouvelle de ces terres, ni y ayant trouvé trace ou reste de notre foi - que la parole du Christ y ait été prêchée avant notre temps ou que cette partie si vaste du monde ait été autrefois découverte ou trouvée par des hommes de notre hémisphère. "

La question fut vivement débattue dans les milieux hérétiques du xvI siècle, qu'une suggestion d'Erasme poussa à conclure que la religion naturelle suffisait au salut. Mais justement, il s'agissait là d'hérétiques et il n'est pas difficile de comprendre pourquoi les ouvrages des deux grands personnages de l'Eglise eurent tant d'importance pour Colomb, qui y trouva un appui pour ses hypothèses: c'était bien un cardinal de l'Eglise, père influent au concile de Constance et chancelier de la Sorbonne, l'évêque Pierre d'Ailly, et par la suite un pontife romain, Pie II, ardent promoteur de la croisade contre le Turc, qui avaient soutenu que les continents étaient circumnavigables et que les antipodes existaient. 
31 C'est donc avec une satisfaction évidente que Colomb souligna au cours de ses lectures les passages qui venaient soutenir ses hyppothèses. Ainsi, dans l'Ymago Mundi, il trouva que Pierre d'Ailly avait repris, sans la réprouver, l'opinion d'Aristote et d'Averroès sur l'étendue des continents par rapport aux mers: elle aurait été bien supérieure, non seulement à celle que la Bible attribuait aux terres émergées, mais aussi à l'estimation de Ptolémée. Celui-ci avait affirmé que la côte occidentale de la péninsule ibérique était distante de 180 degrés de l'extrêmité orientale de l'Asie, c'est-à-dire d'un espace équivalent à la moitié du globe. Or, Pierre d'Ailly rappelait que, d'après le grand philosophe grec, la mer qui sépare l'Espagne de l'Inde était d'une ampleur limitée. Et Colomb glose longuement sur cette page, il mentionne le voyage de Bartholomeu Dias jusqu'à l'extrêmité méridionale de l'Afrique, il souligne que "quod coperitur aqua modicum est ", et quant à la distance des côtes orientales de l'Asie par rapport à celles de l'Occident européen, il écrit : « India est prope Hispaniam ». De même, il avait trouvé dans la Historia de Pie II une confirmation de ses projets : « Auctor docet prout in Germania fuit inventa navis Indorum cum hominibus et mercibus ». Il en concluait : « Si esset maxima distancia non potuissent venisse cum fortuna ». En effet, d'après ce qu'on racontait, le navire s'était échoué à la suite d'une tempête. L'Inde n'était donc pas trop éloignée de l'Allemagne. Et il annotait en marge d'autres récits: «Homines de Catayo versus orientem venierunt. » Ils auraient donc accompli en sens inverse le voyage même qu'il se proposait de faire. Par ailleurs, il était convaincu que des navigateurs étaient déjà arrivés de l'«Arabicus sinus » en parcourant l'Atlantique le long des côtes africaines jusqu'«ad columnas Erculis ».

Dans les pages de Pie II, l'intérêt pour l'hospitalité de la Terre et pour la possibilité de naviguer sur toutes les mers est très marqué. Ainsi, ses descriptions de régions immenses, ses récits de tant de vicissitudes humaines, ou bien ses peintures des moeurs caractérisant les différents peuples du monde, forment une véritable épopée qui ne pouvait pas ne pas séduire un lecteur comme Colomb. L'humaniste siennois était très sensible au paysage et aux beautés naturelles, contemplées cependant avec l'oeil de l'historien passionné: pour lui, ce qui l'environnait n'était pas seulement ce qu'il y discernait immédiatement, mais aussi ce qu'il savait avoir été créé par les hommes.

La force de l'humanisme résidait bien dans sa capacité à se relier étroitement au savoir, aux connaissances des anciens. Comme l'a écrit Alphonse Dupront, « la figure du retour n'est qu'une idée-force, le sens profond d'une attitude créatrice. » Selon lui, l'humanisme fut une époque dépourvue de passé à cause de la cassure qu'il voulut établir avec les siècles qui l'avaient précédé. Toutefois, les esprits novateurs, face au bouleversement des notions acquises, réagirent en puisant dans l'Antiquité de quoi comprendre les nouveautés. Dans les Essais de Montaigne, il y a tout un écheveau de citations classiques qui conduisent assurément à l'homme moderne.

Mais il y avait aussi d'autres voies à parcourir. Après la conquête du Nouveau Monde, ceux que préoccupait la survie des indigènes et qui voulaient en montrer la nature humaine, contre les conquérants et certains écclésiastiques qui les considéraient comme des bêtes, mettaient en lumière leurs formes de vie et leurs croyances comparables à celles de la chrétienté. Il y allait bien sûr d'une tentative de rassurer les lecteurs, et peutêtre même de se rassurer eux-mêmes. Acosta, par exemple, remarque que ces peuples adorent un Dieu, ont des saints à eux et vénèrent même une femme tenant un enfant dans ses bras. Saint François-Xavier découvre, chez les habitants du Japon, une "raison » qui les dispose au baptême. Gonzalez de Mendoza remarque en Chine des communautés 
d'hommes et de femmes qui lui font penser aux monastères catholiques. Cette attitude pouvait apaiser l'inquiétude suscitée par la découverte de peuples qui ignoraient la Révélation, en rétablissant l'unité du genre humain.

Je crois que les certitudes du monde chrétien furent ébranlées plus par les nouveautés de nature anthropologique que par les conséquences scientifiques de la découverte. Les Européens ne paraissent pas avoir été profondément émus par la dilatation soudaine de la Terre - qui doubla en effet - ni par la destruction implicite de la vieille physique, resistant opiniâtrement dans la culture générale. C'est la présence de l'autre - comme l'a bien montré Todorov - qui a troublé les consciences européennes. Heureusement, il y eut des penseurs qui, comme Montaigne, essayèrent de comprendre.

Chez lui aussi cependant, l'émotion se fait sentit: « Nostre monde vient d'en trouver un autre. » Désormais, tout devient possible: «Et qui nous respond si c'est le dernier?» Mais aussi la nature du nouveau monde provoque sa stupeur: "C'estait un monde enfant. » Et néanmoins, en considérant ses habitants, «la plus part de leurs responces et des négociations faictes avec eux tesmoignent qu'ils ne nous devoyent rien en clarté d'esprit naturel et en pertinence.» Lui aussi, le froid Montaigne, malgré son aplomb culturel, débouchant parfois sur le scepticisme, parait bien désireux d'être rassuré par des comparaisons et des similitudes. Mais pas avec la foi et les croyances chrétiennes : son esprit d'humaniste lui fait choisir d'autres vertus. "Quant à la hardiesse et courage, quant à la fermeté, constance, résolution contre les douleurs et la faim et la mort, je ne craindrois pas d'opposer les exemples que je trouverois parmy eux aux plus fameux esemples anciens que nous ayons aus mémoires de nostre monde par deçà.» Son admiration s'enflamme dans cette exclamation célèbre: "Que n'est tombée soubs Alexandre ou soubs ces anciens Grecs et Romains une si noble conqueste!»C'est l'écho de Plutarque et de ses héros qui rententit dans ces mots, exaltant «l'ardeur indomptable ", « la généreuse obstination » d'hommes, de femmes et d'enfants, capables de lutter jusqu'à la mort pour « leurs dieux et leur liberté ». Et ce n'est pas seulement une accusation contre la perfidie espagnole, c'est "nostre monde », le "monde par deçà ", c'est-à-dire la vieille Europe tout entière qui est jugée coupable.

Qu'on relise l'essai du premier livre "Des Cannibales»! L'expérience tragique des guerres de religion avait engendré une sensibilité particulière, une moralité nouvelle chez les meilleurs humanistes français, qui d'ailleurs avaient été moins touchés que les savants d'autres pays par les entreprises liées à la découverte. Un courant oecuménique se développe alors dans la culture française, qui parviendra à formuler le principe de tolérance, seule antidote aux ravages des guerres de religion. La plus noble expression me semble en être le Colloquium heptaplomeres, le Colloque entre sept savants de Bodin, où le débat entre personnages de convictions religieuses différentes se conclut idéalement sur ce verset du psaume: "Ecce quam bonum et quam iucundum cohabitare fratres in unum. » La sincérité de leur foi, la pureté de leurs moeurs, leur sentiment de fraternité, ont permis à ces savants d'arriver à comprendre non seulement les raisons des catholiques et des réformés, des juifs et des musulmans, mais aussi celles des peuples idolâtres d'Amérique.

En même temps, la découverte de sociétés organisées hors d'Europe porte à introduire dans l'histoire l'idée de progrès à la place de la notion de cycle. La lettre d'un historien français, Henri de la Popelinière, envoyée au début du XVII siècle au philologue d'origine italienne Joseph Scaligère, vivant alors aux Pays-Bas, en témoigne. Il y exprimait son dessein de partir pour les pays de l'Orient afin d'en étudier les moeurs et d'essayer de 
comprendre comment «les hommes de sauvages et retirez particuliers qu'on les dicts avoir estés, se sont peu à peu faicts sociaux et unis par divers liens de police humaine. »

Ce n'étaient là que de tout premiers bourgeons d'une culture et d'une mentalité qui ne pouvaient fleurir que bien plus tard. Trop nombreux étaient les freins des différents conservatismes sur le chemin de la civilisation dans le monde même des savants. Il n'en reste pas moins que ce sont là les traces - parfois ténues, parfois consistantes - d'une tradition humaniste au sein de la culture européenne, dont a parlé un grand historien italien, Delio Cantimori, soulignant la force des valeurs, des convictions et des idées qui s'épanouiront en bouquet final à l'âge des Lumières.

\section{NOTES}

1. «Et comme le ciel est subdivisé en deux zones du côté droit, en autant du côté gauche, et en une cinquième, la plus chaude entre toutes, ainsi Dieu se soucia de subdiviser dans le même nombre de zones la masse renfermée par le Ciel, et autant de plages sont imprimées sur la Terre. La médiane est inhabitable à cause de la chaleur; la haute neige couvre les deux autres; entre celles-ci et celle-là, il en plaça deux autres, qu'il rendit tempérées en mêlant ardeur et fraîcheur.»

\section{AUTEUR}

\section{CORRADO VIVANTI}

Professeur à l'Université de Rome-La Sapienza. 